\title{
Application of dynamic indocyanine green perfusion imaging for evaluation of vasoactive effect of acupuncture: a preliminary follow-up study on normal healthy volunteers
}

\author{
This article was published in the following Dove Press journal: \\ Medical Devices: Evidence and Research \\ 20 February 2014 \\ Number of times this article has been viewed
}

Yuri An'

Jong Wook Jeon ${ }^{2}$

Kihwan Kwon ${ }^{3}$

Chulhee Choi'

'Department of Bio and Brain Engineering, KAIST, Daejeon, South Korea; ${ }^{2}$ Acupuncture, Moxibustion and Meridian Research Centre, Korea Institute of Oriental Medicine, Daejeon, South Korea; ${ }^{3}$ Department of Cardiology, College of Medicine, Ewha Womans University, Seoul, Korea
Correspondence: Chulhee Choi Department of Bio and Brain Engineering, KAIST, 29I Daehak-ro, Yuseong-gu,

Daejeon 305-70I, South Korea

Tel 82428694321

Fax $8242-8694380$

Email cchoi@kaist.ac.kr
Background: Even though acupuncture has long been used for alleviating symptoms related to vascular insufficiency, the clinical effect of acupuncture on peripheral circulation has not been fully confirmed. In this study, we investigated whether a near-infrared optical imaging-based method can be used to evaluate the efficacy of the acupuncture procedure to induce changes in peripheral tissue perfusion.

Methods: Two normal, healthy controls were treated with acupuncture on two acupoints (LI-4 and SI-3) three times within 1 week. At the first and third visits, participants were examined using indocyanine green (ICG) perfusion imaging before and 10 minutes after the acupuncture procedure. Blood perfusion of the hands was determined after intravenous bolus injection of ICG and dynamic analysis of the fluorescence signals by near-infrared imaging system.

Results: The blood perfusion rates of the hands were markedly increased immediately after acupuncture at the first trial in both cases. The baseline perfusion rates of the hands measured at the third visit were higher compared to the original basal level in one case; there was no difference in baseline perfusion rates of both hands in another case. In both cases, there was no acute effect of acupuncture on hand perfusion at the third trial.

Conclusions: These results collectively suggest a potential of the ICG perfusion imaging as an effective evaluation tool to validate the vasoactive effect of acupuncture.

Keywords: vasodilation, blood perfusion, imaging, Raynaud syndrome

\section{Introduction}

Acupuncture has been a component of the health care system in Asia for at least 2,500 years. ${ }^{1}$ It also has been introduced in the US and practiced widely as an alternative medicine. However, the basic principles of acupuncture are still hypothetical and unknown. ${ }^{2}$ Hence, it is relevant and significant to study how acupuncture works and measure the quantitative effect of acupuncture in various clinical settings.

Many studies have shown beneficial effects of acupuncture on tissue perfusion by showing correlation between cutaneous blood flow and acupuncture ${ }^{3}$ or measuring changes in regional cerebral blood flow and glucose metabolism following electroacupuncture. ${ }^{4}$ There are substantial numbers of trials to demonstrate the peripheral vasomotor effect of acupuncture..$^{2,5,6}$ To measure the peripheral vasomotor activity, laser Doppler imaging (LDI) has been widely used., ${ }^{2,6}$ Even though LDI is easy to use and noninvasive, the information provided by the method is not quantitative and therefore cannot be used for longitudinal or comparison studies. ${ }^{7}$ 
We have recently developed a method enabling quantitative measurement of tissue blood perfusion rate using indocyanine green (ICG) as a dye. ${ }^{7}$ Using this method, peripheral tissue perfusion can be measured quantitatively and sensitively compared to the other conventional methods such as LDI. ${ }^{7}$ This optical imaging-based technique can also be applied for differential diagnosis of peripheral vascular insufficiencies, including peripheral arterial occlusive diseases and functional Raynaud syndrome..$^{8-10}$ In the present study, we tested whether this method can be used for evaluating the vasoactive effect of acupuncture.

\section{Methods}

\section{Volunteers}

Two healthy volunteers, a 39-year-old male and a 20-year-old female, participated in this study. Neither participant was treated with any therapeutic medication during the study. They had no cardiovascular risk factors or symptoms related to peripheral vascular insufficiency. The study protocol was approved by the institutional review board of Mokdong Medical Center (Seoul, South Korea).

\section{Acupuncture}

All acupuncture procedures were done by an experienced specialist in acupuncture having more than 5 years of clinical practice. The choice of acupoints was based on their specific character based on traditional text. Hegu (LI-4) is believed to have the function of speeding blood circulation of upper extremities. It increases blood perfusion. ${ }^{2}$ Also, there was a positive influence of acupuncture treatment in pregnant women when using a combination of SP-6 (Sanyinjiao) and LI-4 (Hegu) acupuncture points. ${ }^{11}$ Houxi (SI-3) is believed to soothe hand numbness (Figure 1A). Those two acupoints are generally used, and have an effect to increase blood perfusion. ${ }^{2,11,12}$

Both acupoints were sterilized with ethanol, and stainless needles $(0.25 \times 40 \mathrm{~mm})$, also sterilized, were inserted $1.5-2.0 \mathrm{~cm}$ and manipulated for 10 seconds until deqi sensation was achieved; they then remained untouched. After 10 minutes, the needles were taken out without manipulation.

On the first acupuncture treatment day, subjects were asked to wait for 10 minutes to adjust to the room atmosphere, and the imaging was performed simultaneously with the injection of ICG solution via catheter on median basilic vein according to our customized ICG imaging model. Imaging takes 15 minutes or so; after imaging, acupuncture was performed for 10 minutes. Another imaging was performed 10 minutes after acupuncture to test the acute effect of acupuncture on the perfusion rate in the hands. On the second acupuncture treatment day, acupuncture was performed without ICG imaging. The process of the first day was repeated on the third acupuncture treatment day (Figure 1C).
A
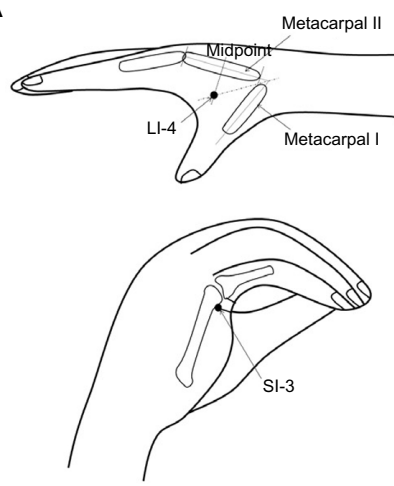

C

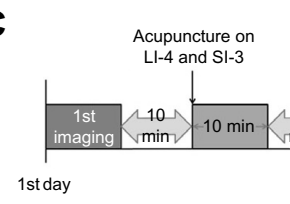

B

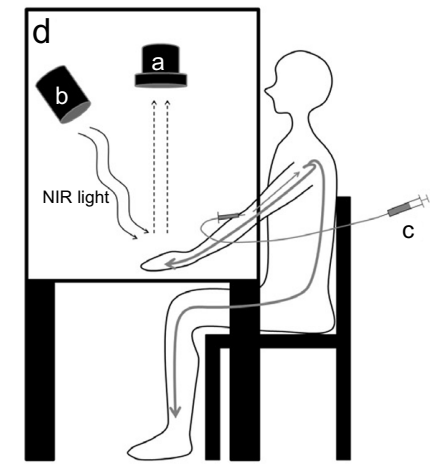

a: CCD camera

b: IR emitter

c: ICG catheter

injection

d: darkroom

Figure I Study protocol for acupuncture and ICG perfusion imaging.

Notes: (A) Acupoints (LI-4 and SI-3) used for the current study are illustrated. (B) Near infrared optical imaging setting for ICG fluorescence is shown. Hands of the subject should be rested in the dark box during the acquisition of ICG fluorescence signals via CCD camera. (C) The study protocol for acupuncture and ICG perfusion imaging. On the first day, the ICG perfusion imaging was performed before and after acupuncture. On the second visit, acupuncture was performed without ICG imaging. The process of the first day was repeated on the third acupuncture treatment day.

Abbreviations: CCD, charge-coupled device; ICG, indocyanine green; IR, infrared; NIR, near-infrared. 


\section{ICG imaging and analysis}

For ICG time-series imaging, 40 to 80 images $(768 \times 512$ pixels $)$ of the dorsum of both hands were taken at 5 -second intervals for 5 minutes immediately after an intravenous bolus injection of ICG $(0.16 \mathrm{mg} / \mathrm{kg})$ using a near-infrared (NIR) imaging system including two $760 \mathrm{~nm}$ LED arrays and a charge-coupled device with $830 \mathrm{~nm}$ long-pass filter (Vas View, Vieworks Corp, Seongnam, Gyeonggi-do, South Korea) (Figure 1B), as used for previous studies..$^{8-10}$ Regional ICG dynamics obtained from each pixel were analyzed to get the perfusion rate and $\mathrm{T}_{\max }$ value. Perfusion rate $(\% /$ minute) was defined as the fraction of blood exchanged per minute in the vascular volume of the region of interest. ${ }^{7} \mathrm{~T}_{\max }$ was defined as the time from ICG injection to peak time of ICG dynamics. ${ }^{7}$ To visualize the segmental pattern of ICG dynamics, the perfusion rate and $T_{\text {max }}$ value of each pixel in the hand was calculated and translated into a pseudocolor-coded perfusion rate and $\mathrm{T}_{\max }$ map using Visual C++ (2008 version, Microsoft Corporation, Redmond, WA, USA), as analyzed in previous studies. ${ }^{8-10}$

\section{Results and discussion}

To test the effect of acupuncture on LI-4 and SI-3 on blood perfusion, volunteers were subjected to serial ICG perfusion imaging before and immediate after acupuncture on the first and third visits. The average values of the perfusion rate were calculated for the fingertips, which are the first segment of perfusion map and analyzed for the effect of acupuncture (Table 1). In the female subject, the baseline perfusion rate was lower compared to the male subject. Even though there is considerable difference in baseline perfusion rates, the acupuncture treatment significantly increased the blood perfusion in both cases on the first visit (Figure 2A and B). However, such acute effects were not observed on the third trial. On the contrary, the chronic effect of acupuncture was only observed in the female subject. The three trials of acupuncture increased the baseline blood perfusion up to 2.5 -fold in the female; there was no significant difference in baseline perfusion in the male subject. The acute and chronic effects of acupuncture are in good concordance with the previous studies using other conventional methods. ${ }^{2,11,12}$ These results might indicate the beneficial effect of acupuncture on peripheral vascular function.

Acupuncture has also been used for treatment of functional vascular insufficiencies such as Raynaud syndrome, even though the efficacy is still undetermined. ${ }^{13}$ Since Raynaud syndrome is quite common in young females, we analyzed the $T_{\text {max }}$ kinetics of both subjects (Figure $2 \mathrm{C}$ and $\mathrm{D}$ ). As the perfusion map, the $\mathrm{T}_{\max }$ value was also color-coded in relative linear scale. Segmental analysis of $T_{\text {max }}$, the time-to-peak from ICG injection, can provide information regarding peripheral vascular function. ${ }^{8}$ Both volunteers showed the normal pattern of $\mathrm{T}_{\max }$ map. Even though the female showed lower baseline perfusion, the $\mathrm{T}_{\text {max }}$ analysis clearly demonstrated that both cases were free from Raynaud syndrome. We could not find any significant changes in $\mathrm{T}_{\max }$ kinetics in both cases by acupuncture (data not shown).

Even though the current study employed only two cases, this pilot study clearly demonstrated the potential of the ICG perfusion imaging for quantitative and reproducible evaluation of acupuncture effects on peripheral vasculature. There was considerable effect of acupuncture treatment in both acute and chronic manner. However, the lack of acute acupuncture effect on the repeated trials should be further evaluated in a large number of subjects. Another issue is the variation in chronic effect of acupuncture. The reason why the repeated treatment of acupuncture had chronic beneficial effect in the female case might be due to the lower baseline perfusion (on the first visit). Even though the baseline level of the subject was not low enough to be diagnosed as peripheral vascular insufficiency, there might be more room for improvement in cases with relatively poor perfusion. One of the advantages of the ICG perfusion for evaluation of vasomotor activity of acupuncture is that the method can provide information regarding not only vascular perfusion but also functional integrity of peripheral vasculature. ${ }^{8}$ Therefore, the effect of acupuncture on Raynaud syndrome can also be tested as previously reported. ${ }^{14}$

Table I The effect of acupuncture on the blood perfusion rate

\begin{tabular}{|c|c|c|c|c|c|c|c|}
\hline \multirow[t]{2}{*}{ Case } & \multirow[t]{2}{*}{ Hand } & \multicolumn{3}{|l|}{ First visit } & \multicolumn{3}{|l|}{ Third visit } \\
\hline & & $\begin{array}{l}\text { Baseline perfusion } \\
(\% / \mathrm{min})\end{array}$ & $\begin{array}{l}\text { Postacupuncture } \\
\text { (\%/min) }\end{array}$ & $\begin{array}{l}\text { Change } \\
\text { (\%) }\end{array}$ & $\begin{array}{l}\text { Baseline perfusion } \\
(\% / \mathrm{min})\end{array}$ & $\begin{array}{l}\text { Postacupuncture } \\
\text { (\%/min) }\end{array}$ & $\begin{array}{l}\text { Change } \\
(\%)\end{array}$ \\
\hline Case I & Right & 99.6 & 124.4 & 24.9 & 95.0 & 97.8 & 3.0 \\
\hline (male, 39) & Left & 83.5 & 119.5 & 43.1 & 79.9 & 80.8 & I.I \\
\hline Case 2 & Right & 52.9 & 72.2 & 36.6 & 123.8 & 131.7 & 6.4 \\
\hline (female, 20) & Left & 45.0 & 64.9 & 44.3 & 114.7 & 110.6 & -3.6 \\
\hline
\end{tabular}


A

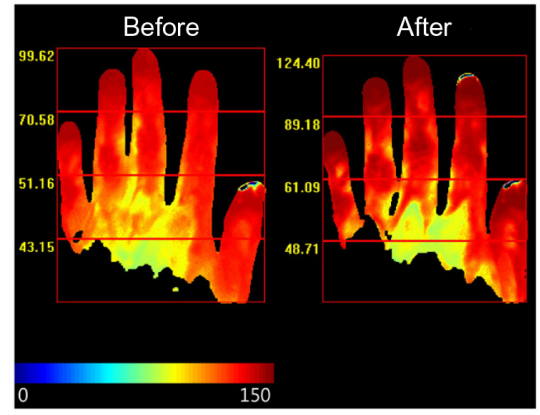

C

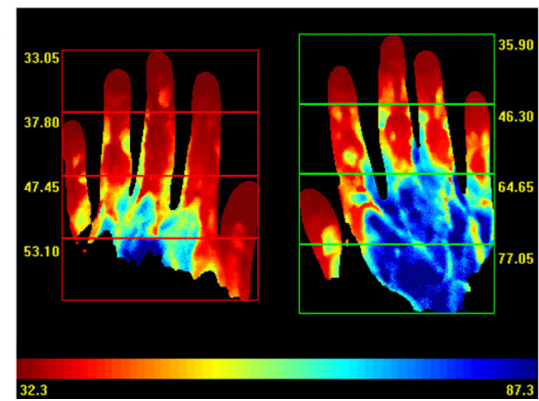

B

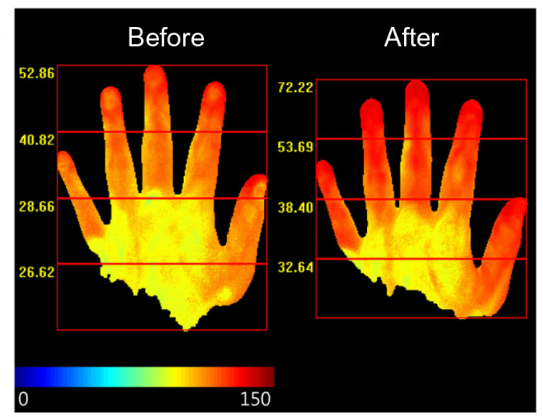

D

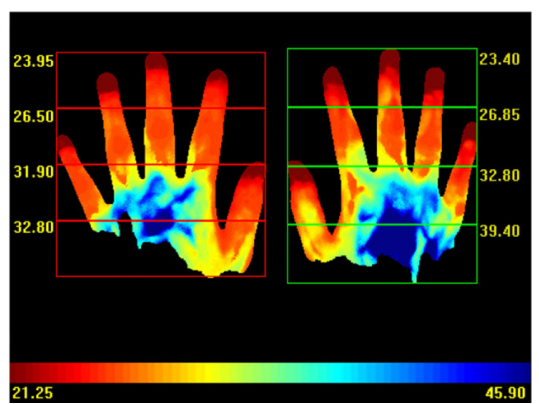

Figure 2 The effect of acupuncture on blood perfusion.

Notes: (A) A representative figure shows the blood perfusion of a healthy 39-year-old male before and 10 minutes after acupuncture on the first visit. The blood perfusion rate of the hands was shown as a two-dimensional pseudocolored map. At each pixel point of $A$ and $B$, the calculated blood perfusion rate is color-coded; red indicates good perfusion, while blue corresponds to poor perfusion. It is in log scale, and it indicates from the minimum value 0 to a maximum value of I50. In both figures, the left side hands show the blood perfusion rate map before applying acupuncture, and the right ones show after acupuncture. (B) A representative figure shows the blood perfusion of a healthy 20-year-old female before and 10 minutes after acupuncture on the first visit. (C and $\mathbf{D})$ Color-coded maps indicate $T_{\text {max }}$ values of the hands of two normal subjects. For sequential analysis of $T_{\max }$, the dorsum of the hand was divided into four identical squares, and the average value of $T_{\max }$ was measured. The value that is written next to the square represents the average value of perfusion rate or $\mathrm{T}_{\max }$ of each square.

\section{Disclosure}

This research was supported by a National Research Foundation grant funded by the Korea government (MEST) (No. 2012M3A9C5048707). The authors report no other conflicts of interest in this work.

\section{References}

1. Acupuncture. NIH Consens Statement. 1997;15(5):1-34.

2. Zhang W-b, Wang L-1, Huang T, et al. Laser doppler perfusion imaging for assessment of skin blood perfusion after acupuncture. Med Acupunct. 2008;20(2):109-118.

3. Jansen G, Lundeberg T, Kjartansson J, Samuelson UE. Acupuncture and sensory neuropeptides increase cutaneous blood flow in rats. Neurosci Lett. 1989;97(3):305-309.

4. An YS, Moon SK, Min IK, Kim DY. Changes in regional cerebral blood flow and glucose metabolism following electroacupuncture at LI 4 and LI 11 in normal volunteers. J Altern Complement Med. 2009;15(10): 1075-1081.

5. Birch S, Hesselink JK, Jonkman FA, Hekker TA, Bos A. Clinical research on acupuncture: Part 1 . What have reviews of the efficacy and safety of acupuncture told us so far? J Altern Complement Med. 2004;10(3): 468-480.

6. Litscher G, Wang L, Huber E, Nilsson G. Changed skin blood perfusion in the fingertip following acupuncture needle introduction as evaluated by laser Doppler perfusion imaging. Lasers Med Sci. 2002;17(1): $19-25$.
7. Kang Y, Choi M, Lee J, Koh GY, Kwon K, Choi C. Quantitative analysis of peripheral tissue perfusion using spatiotemporal molecular dynamics. PLoS One. 2009;4(1):e4275.

8. Kang Y, Lee J, An Y, Jeon J, Choi C. Segmental analysis of indocyanine green pharmacokinetics for the reliable diagnosis of functional vascular insufficiency. J Biomed Opt. 2011;16(3):030504.

9. Kang Y, Lee J, Kwon K, Choi C. Application of novel dynamic optical imaging for evaluation of peripheral tissue perfusion. Int $J$ Cardiol. 2010;145(3):e99-e101.

10. Kang Y, Lee J, Kwon K, Choi C. Dynamic fluorescence imaging of indocyanine green for reliable and sensitive diagnosis of peripheral vascular insufficiency. Microvasc Res. 2010;80(3):552-555.

11. Zeisler H, Eppel W, Husslein P, Bernaschek G, Deutinger J. Influence of acupuncture on Doppler ultrasound in pregnant women. Ultrasound Obstet Gynecol. 2001;17(3):229-232.

12. Naruse S, Mori K, Kurihara M, et al. [Chorioretinal blood flow changes following acupuncture between thumb and forefinger]. Nihon Ganka Gakkai Zasshi. 2000;104(10):717-723. Japanese.

13. Malenfant D, Catton M, Pope JE. The efficacy of complementary and alternative medicine in the treatment of Raynaud's phenomenon: a literature review and meta-analysis. Rheumatology (Oxford). 2009;48(7):791-795.

14. Donoyama N, Ohkoshi N. Electroacupuncture therapy for arthralgia and Raynaud's phenomenon in a patient with systemic lupus erythematosus. Acupunct Med. 2010;28(1):49-51. 
Medical Devices: Evidence and Research

Dovepress

\section{Publish your work in this journal}

Medical Devices: Evidence and Research is an international, peerreviewed, open access journal that focuses on the evidence, technology, research, and expert opinion supporting the use and application of medical devices in the diagnosis, treatment and management of clinical conditions and physiological processes. The identification of novel devices and optimal use of existing devices which will lead to improved clinical outcomes and more effective patient management and safety is a key feature. The manuscript management system is completely online and includes a quick and fair peer-review system. Visit http://www. dovepress.com/testimonials.php to read real quotes from authors.

Submit your manuscript here: http://www.dovepress.com/medical-devices-evidence-and-research-journal 